\title{
The Clinical and Radiological Availability of Percutaneous Balloon Kyphoplasty as a Treatment for Osteoporotic Burst Fractures
}

\author{
Ki Chan An, SukJung Kang, Jang Suk Choi, Jin Hyuk Seo \\ Department of Orthopedic Surgery, Busan Paik Hospital, College of Medicine, Inje University, Busan, Korea
}

\begin{abstract}
Study Design: We retrospectively assessed the results of percutaneous balloon kyphoplasty (KP) by clinical and radiological methods.

Purpose: To evaluate the outcome of KP as a treatment for osteoporotic burst fractures.

Overview of Literature: Many surgeons are concerned about the possibility of neurological complications after percutaneous kyphoplasty for osteoporotic burst fractures, secondary to intra-canal cement leakage.

Methods: We performed KP as a treatment for osteoporotic burst fractures. We studied 12 patients/13 vertebrae. The two control groups consisted of patients who only underwent conservative treatment and those who underwent posterior instrumentation and fusion. We measured each preoperative/postoperative vertebral kyphotic deformity angle (KDA) using simple lateral spine images and checked for leakage of cement, as well. The preoperative/postoperative visual analog scale (VAS) scores for back pain, degree of daily activity, and postoperative complications were evaluated.

Results: The mean improvement in KDA after KP was $9.7 \pm 2.2^{\circ}$. The mean preoperative and postoperative VAS scores for back pain were $8.3 \pm 0.4$ and $3.1 \pm 0.17$, respectively. Regarding the control group, the mean postoperative VAS score for the conservative group and the posterior surgery group decreased by $4.5 \pm 0.17$ and $3.2 \pm 0.19$, respectively. There was no statistically significant difference between the KP and posterior surgery groups $(\mathrm{p}=0.125$ ). However, there was a statistically significant difference between the KP and conservative treatment groups $(\mathrm{p}=0.012)$.

Conclusions: KP is safe and useful for treating osteoporotic burst fractures.
\end{abstract}

Key Words: Thoracolumbar spine, Osteoporotic burst fracture, Percutaneous balloon kyphoplasty, Cement leakage

\section{Introduction}

Vertebroplasty and kyphoplasty (KP) are very effective treatments for osteoporotic fractures involving the anterior column of the spine. Many surgeon are concerned about the possibility of neurological complications secondary to intracanal cement leakage, and they hesitate to perform percutaneous vertebroplasties and KPs for osteoporotic burst fractures. Generally, in the case of burst fractures, conservative treatment and posterior instrumentation with bone grafting are popular treatment options. Unlike other methods, however, there is no evidence to suggest that conservative treat- ment improves pain and ambulation. We examined the clinical and radiological outcomes in patients undergoing percutaneous KP as a treatment for osteoporotic burst fractures refractory to 4 weeks of conservative treatment.

\section{Materials and Methods}

Between January 2004 and April 2006, we performed percutaneous balloon KP as a treatment for osteoporotic burst fractures in 13 vertebrae (12 senile patients, all women; age range 66 84 years; mean age, 78 years). Minimal follow-up duration was over 1 year. Magnetic reso-

Corresponding author: SukJung Kang, MD

Department of Orthopedic Surgery, Busan Paik Hospital, Inje University

\#633-165, Gaegeum-dong, Busanjin-gu, Busan, 614-735, Korea

Tel: +82-51-890-6129, Fax: +82-51-892-6619, E-mail: orthosj@yahoo.co.kr

This work was supported by Inje University Grant of 2007 years. 
nance imaging (MRI) and computed tomography (CT) scans were obtained to evaluate for the presence of acute vertebral fractures in each patient's spine. They were also used to assess the relative degree of continuity of the posterior vertebral wall and the degree of canal encroachment, and to eliminate any other source of pain. All patients had a stable burst fracture pattern. We excluded patients with neurological symptoms and those with posterior column invasion.

These osteoporotic burst fractures had relatively acute findings on MRI or CT images. Patients complained of initially experiencing difficulty in daily activities and walking and having severe tenderness around the vertebral fracture site. The patients were very old and had severe osteoporosis (under -4.6 bone mineral density [BMD] T score), along with other medical comorbidities. They were treated with bed rest, analgesics, and braces over the course of 4 weeks. However, they still complained of back pain after conservative treatment. We assumed that the incidence of cement leakage would be greater before the 4-week mark, and the optimal time to correct kyphotic deformity was considered to be 4 weeks after burst fracture.

Our control group consists of 33 cases of conservative treatment and 13 cases of posterior instrumentation and bone fusion (conservative treatment group: age range 64 88 years; mean age, 80 years; and under -4.7 BMD T score; posterior surgery group: age range 60 to 81 years; mean age, 74 years; and under -4.2 BMD T score). These patients were also followed for over 1 year. There were no statistical differences between the study group and the control group with respect to age and BMD ( $\mathrm{p}=0.153)$. We retrospectively compared the percutaneous balloon KP group with the control group. We performed 5 thoracic and 8 lumbar procedures (Table 1). Five patients had diabetes, and three

Table 1. Location of osteoporotic burst fracture

\begin{tabular}{lc}
\hline T12 & 5 \\
L1 & 6 \\
L2 & 2 \\
\hline Total & 13 \\
\hline
\end{tabular}

patients had cardiovascular disease. Moreover, one patient had chronic obstructive pulmonary disease. Both diabetes and cardiovascular disease were observed in three patients. Preoperative BMD revealed the presence of osteoporosis in 12 patients ( $\mathrm{T}$ score range, -6.2 to -4.6 ; mean $\mathrm{T}$ score -4.9 ) (Table 2).

After skin infiltration with local anesthesia, a $1 \mathrm{~cm}$ paramedian incision was made. On anteroposterior fluoroscopy, an 11-gauge bone biopsy needle was centered over the pedicle at the 10 o'clock (left pedicle) and 2 o'clock (right pedicle) positions. The needle was medialized through the cylinder of the pedicle to reach the middle of the vertebral body. A guide pin was passed through the biopsy needle into the vertebral body. The needle was then removed, and the access portal was expanded with larger cannulae. Through this cannula, an inflatable balloon was advanced. Once both balloons had been inserted, they were sequentially inflated until the fracture was realigned or maximal inflation pressure (220 psi) had been reached. The balloons were then removed, and the bone was stabilized with polymethyl methacrylate.

We radiologically assessed the kyphotic deformity angle (KDA), which was defined as the measured angle between the upper end plate and the lower end plate of the fractured vertebra. We also looked for clefts within the fractured vertebra and searched for cement leakage into the posterior spinal canal in the pre-operative and post-operative planes on anteroposterior and lateral radiographs. We assessed clinical symptoms by asking patients to quantify their degree of pain on the visual analog scale (VAS) before KP, on the third day after KP, 6 months after KP, and 1 year after KP. Mobility was evaluated using Chen and Lee's semiquantitative scale: 0 , walking without assistance; 1 , walking with assistance; 2 , wheelchair-bound; 3 , activity

Table 2. Bone mineral density ( $\mathrm{T}$ score)

\begin{tabular}{lr}
\hline$-4.5 \sim-4.9$ & 6 \\
$-5.0 \sim-5.4$ & 4 \\
$-5.5 \sim-5.9$ & 1 \\
$-6.0 \sim-6.5$ & 1 \\
\hline Total & 12 \\
\hline
\end{tabular}

Table 3. Radiological results of kyphoplasty

\begin{tabular}{lccc}
\hline & Pre Op & Post Op & p-value \\
\hline Kyphotic angle & $15.9 \pm 2.4$ degree & $6.2 \pm 1.6$ degree & 0.016 \\
\hline
\end{tabular}

Wilcoxon signed ranks test, $\mathrm{p}<0.05$. 
restricted to sitting in bed; 4, activity restricted to laying flat in bed ${ }^{1}$. Statistical analysis was performed using the Wilcoxon signed ranks test.

\section{Results}

The overall KP results were auspicious. After completion of the procedure, the KDA improved significantly. Before balloon kyphoplasty, the mean KDA was $15.9 \pm 2.4^{\circ}$ for stable burst fractures. After the procedure, the mean KDA was $6.2 \pm 1.6^{\circ}$, showing an improvement of $9.7 \pm 2.2^{\circ}$ (Table 3). At final follow-up, the KDA was $5.9 \pm 1.4^{\circ}$. The mean KDAs in the conservative treatment group at initial presentation and at final follow-up were $5.2 \pm 1.4^{\circ}$ and 14.8 $\pm 2.1^{\circ}$, respectively. There was a statistically significant difference between the KP and conservative treatment groups with regard to improvement in $\mathrm{KDA}(\mathrm{p}=0.016)$. The mean KDA of the open posterior surgery group before and after the procedure was $19.1 \pm 2.4^{\circ}$ and $9.1 \pm 1.8^{\circ}$, respectively; the final follow-up KDA was $8.9 \pm 1.7^{\circ}$. Therefore, there was no statistically significant difference between the $\mathrm{KP}$ and open posterior surgery groups with regard to the improvement in KDA ( $\mathrm{p}=0.081$ ). No patients had significant complications related to cement leakage into the posterior spinal canal (Fig. 1). One patient had cement leakage into the intervertebral disc secondary to an upper end-plate fracture, but the patient did not have neurologic symptoms (Fig. 2).

The reductions in pain from baseline to the third day and to 3 months post-operative were statistically significant (VAS scores: pre-operative, $8.3 \pm 0.4 ; 3$ days post-operative, $3.9 \pm 0.2 ; 3$ months post-operative, $3.2 \pm 0.17$; 1 year post-operative, $3.1 \pm 0.17)$. The average VAS score decreased by $5.2 \pm 0.3$. Every patient was capable of walking autonomously one day after KP. After conservative
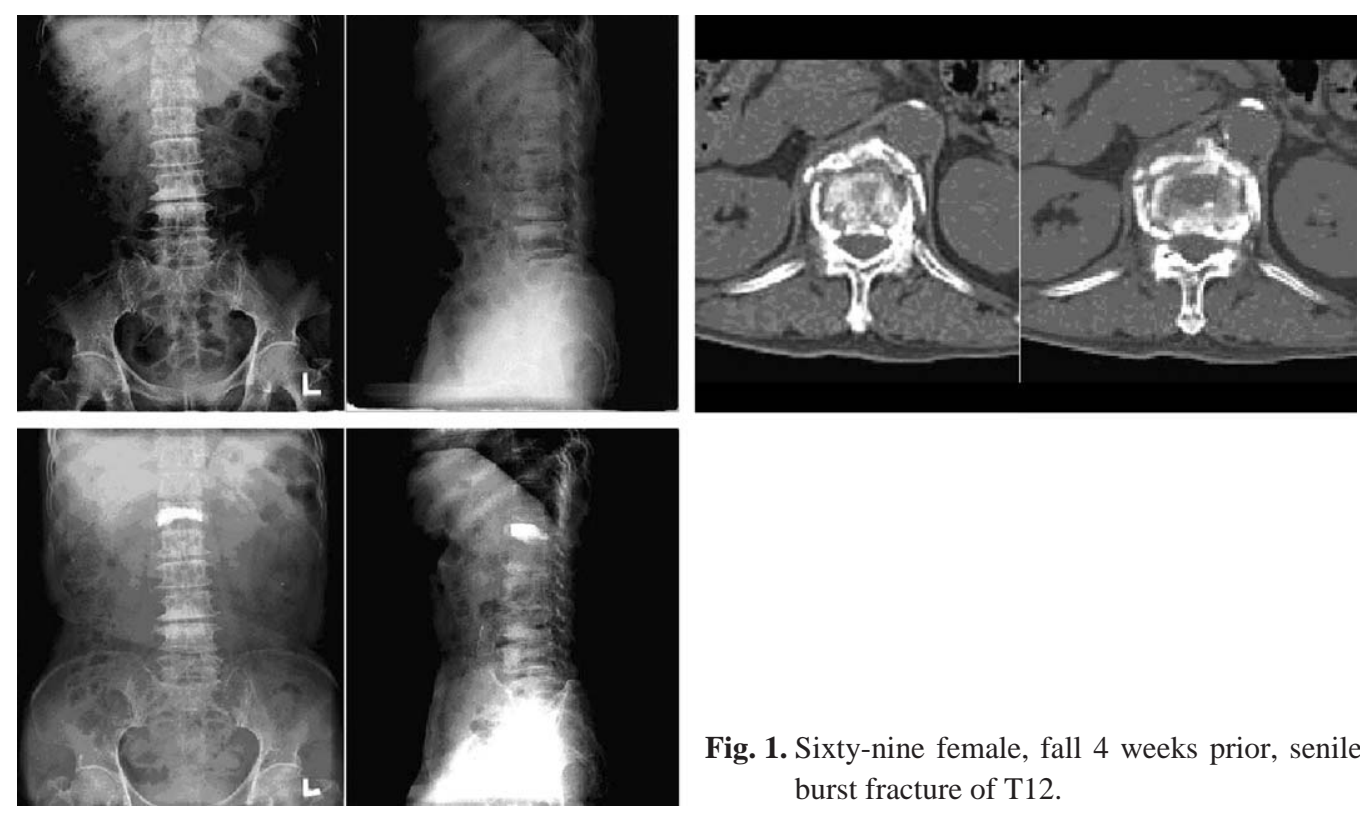

Fig. 1. Sixty-nine female, fall 4 weeks prior, senile burst fracture of $\mathrm{T} 12$.

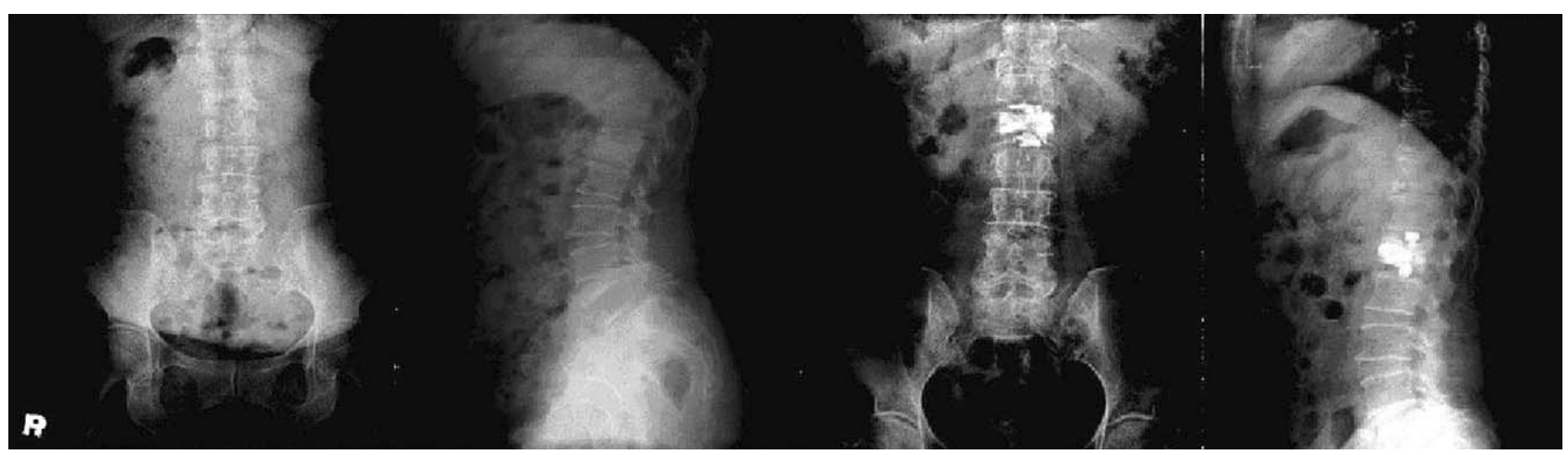

Fig. 2. Sixty-three female, fall 10 weeks prior, senile burst fracture of L1. 
treatment, the VAS score decreased by $3.4 \pm 0.2$ (VAS score: pre-operative, $7.9 \pm 0.4$; after 3 months, $4.8 \pm 0.18$; after 1 year, $4.5 \pm 0.17$ ). Hence, there was a statistically significant difference between the KP and conservative treatment groups with regard to improvement in the VAS score $(\mathrm{p}=0.012)$. After posterior instrumentation and fusion, the VAS score decreased by $5.3 \pm 0.3$ (VAS score: pre-operative, $8.5 \pm 0.5 ; 3$ days post-operative, $3.5 \pm 0.21 ; 3$ months post-operative, $3.4 \pm 0.2$; 1 year post-operative, $3.2 \pm 0.19$ ). Hence, there was no statistically significant difference between the KP and open posterior surgery groups with regard to improvement in VAS score $(\mathrm{p}=0.125)$.

The initial Chen and Lee semi-quantitative scale was grade 3 or 4 in all patients. In the KP group, 12 patients (100\%) improved to grade 0 at final follow-up. In the conservative treatment group, 12 patients (36.5\%) improved to grade 0,18 patients $(54.5 \%)$ improved to grade 1 , and 3 patients (9\%) improved to grade 2 at final follow-up. In the posterior instrumentation and fusion group, 13 patients (100\%) improved to grade 0 at final follow-up.

The average volume of bone cement injected was $5 \mathrm{cc}$. No patient complained of symptoms suggesting pulmonary embolism. Postoperative CT scans never revealed cement leakage.

\section{Discussion}

The two most common treatment methods for osteoporotic burst fractures are conservative treatment and posterior instrumentation and fusion. Conservative treatment can be performed in senile patients with medical comorbidities; however, conservative treatment may lead to cardiovascular complications, as well as decreased correction rate for kyphotic deformities. Posterior instrumentation and fusion provides solid stability; however, posterior instrumentation and fusion is not a viable surgical option for patients who lack sufficient bone mass from osteoporotic burst fractures. Age and comorbid medical conditions may further contribute to the impracticality of posterior instrumentation and fusion as a treatment for osteoporotic burst fractures.

$\mathrm{KP}$, as well as vertebroplasty, offers significant pain relief and fracture stabilization. The application of percutaneous vertebroplasty as a minimally invasive procedure is popular and pervasive. Nevertheless, the danger of cement leakage into the posterior spinal canal has precluded percutaneous vertebroplasty as a treatment for burst fractures. In 2004, however, Chen and Lee', reported a clinically satisfactory result by performing percutaneous vertebroplasty in six stable burst fractures without osteoporosis. Although cement leakage into the adjacent intervertebral disc space occurred in four instances $(66.7 \%)$, it did not cause the neurological symptoms associated with cement leakage into the posterior spinal canal. Despite its evident benefits with regard to pain relief and vertebral stabilization, vertebroplasty is not as effective in correcting kyphotic deformities. Balloon KP was developed in order to compensate for vertebroplasty's disadvantages in correcting kyphotic deformities. Balloon KP minimizes the possibility of cement leakage. Hence, we performed balloon KP for the treatment of osteoporotic burst fractures.

Balloon KP was developed by applying Dr. Mark Reiley's balloon tamp in percutaneous vertebroplasty ${ }^{2,3}$. The balloon tamp was developed by Reiley in 1997, with the goal of restoring vertebral body height and reducing kyphosis. KP involves the inflation of a balloon tamp before injection of bone cement in the compressed vertebral body, in an effort to reduce kyphotic deformity. Shindle et al. ${ }^{4}$, reported that balloon KP enhances the height reduction $>4.5$-fold over the positioning maneuver alone and accounts for over $80 \%$ of the ultimate reduction. Because KP enables the injection of bone cement at low pressure, the procedure minimizes the possibility of cement leakage ${ }^{5-7}$.

Reportedly, the complication rate following balloon KP is similar to that seen in vertebroplasty. Complications deriving from vertebroplasty as a treatment for osteoporotic burst fracture occur in less than $1 \%$ of patients when the procedure is performed by an experienced surgeon. The complication rate stemming from vertebral pathologic compression fractures is 5 10\%. Although complications occur slightly more frequently in KP than in vertebroplasty, we believe that such results derive from KP's relative dearth of recognition. However, according to Orlando, the complication rate with respect to symptomatic cement extravasation may be lower in KP than in vertebroplasty ${ }^{2}$. We observed only one instance of cement leakage in this study. We attribute our success to 4 weeks of conservative treatment prior to the procedure and our decision to perform KP in stable burst fractures. Hulme et al. ${ }^{8}$, reported the following distribution of cement extravasation in vertebroplasty: epidural, $32 \%$; paraspinal, $32.5 \%$; intradiscal, $30.5 \%$; pulmonary, $1.7 \%$; and foraminal, $3.3 \%$. He further reported the following distribution of cement extravasation in KP: epidural, $11 \%$; paraspinal, $48 \%$; intradiscal, $38 \%$; pulmonary, $1.5 \%$; 
and foraminal, 1.5\%. In most cases (vertebroplasty, 96\%; KP, $89 \%$ ), symptoms do not accompany cement extravasation. Intradiscal cement leakage, which occurs relatively frequently, may cause mechanical overload to the disc and/or to the adjacent vertebrae. Lin et al. ${ }^{9}$, reported that $58 \%$ of vertebral bodies adjacent to a disc with cement leakage fractured during the follow-up period, compared with $12 \%$ of vertebral bodies adjacent to a disc without cement leakage after vertebroplasty. We only observed complications accompanying cement leakage in a single case.

Frail older patients have poor tolerance for the bed rest required in non-operative management and the physiologic stress engendered by an open stabilization procedure ${ }^{10}$. Indications for open surgery are rare and include major neurologic injury and mechanical instability. Anterior decompression is an effective method; however, the morbidity rate and the risk of spinal deterioration in instrumentation and fusion are very high in senile patients. Posterior decompression and stabilization are perhaps better tolerated by older patients. The pedicles remain stronger than the vertebral bodies, but implant loosening remains a problem ${ }^{10}$.

We did not observe any major complications when we performed KP as a treatment for stable burst fractures. The clinical and radiological results of KP were superior to those of conservative treatment. Lyritis et al. ${ }^{11}$ showed that pain decreased by $33 \%$ at day 14 in vertebral compression fracture patients treated with the conservative method, and Gennari et al. ${ }^{12}$, noted that pain decreased by $40 \%$ at day 30 . However, Silverman ${ }^{13}$ noted the long-term sequelae associated with vertebral compression fractures: chronic back pain, muscle fatigue, reduced exercise tolerance, early satiety, insomnia, fibromyalgia, and low self-esteem. Therefore, vertebral fractures must be treated aggressively. The results of conservative treatment seen in this study were inferior to those seen in previous studies. However, the previous studies addressed compression fractures, while we assessed burst fractures. We attained very satisfactory results with $\mathrm{KP}$, in comparison to those attained with posterior instrumentation and fusion. The limitation of our study lies in the insufficient number of cases and short follow-up duration. Future studies are recommended.

\section{Conclusions}

Osteoporotic burst fractures occur most frequently at the thoracolumbar junction. Percutaneous vertebroplasty and balloon KP are minimally invasive treatments for osteoporotic burst fractures. However, the danger of cement leakage into the posterior spinal canal has precluded some surgeons from performing these two procedures.

Nevertheless, balloon KP is somewhat beneficial for strengthening the spinal wall. Because KP enables the injection of bone cement at low pressure, the procedure further diminishes the possibility of cement leakage ${ }^{5-7}$.

Balloon KP is useful in correcting deformities, alleviating pain, and ameliorating immobility, and it is a practical treatment for osteoporotic burst fractures.

\section{REFERENCES}

1. Chen JF, Lee ST: Percutaneous vertebroplasty for treatment of thoracolumbar spine bursting fracture. Surg Neurol 2004; 62: 494-500.

2. Ortiz AO, Zoarski GH, Beckerman M: Kyphoplasty. Tech Vasc Interv Radiol 2002; 5: 239-249.

3. Lieberman IH, Dudeney S, Reinhardt MK, Bell G: Initial outcome and efficacy of "kyphoplasty" in the treatment of painful osteoporotic vertebral compression fractures. Spine 2001; 26: 1631-1638.

4. Shindle MK, Gardner MJ, Koob J, Bukata S, Cabin JA, Lane JM: Vertebral height restoration in osteoporotic compression fractures: kyphoplasty balloon tamp is superior to postural correction alone. Osteoporos Int 2006; 17: 1815-1819.

5. Garfin SR, Yuan HA, Reiley MA: New technologies in spine: kyphoplasty and vertebroplasty for the treatment of painful osteoporotic compression fractures. Spine 2001; 26: 1511-1515.

6. Hardouin P, Fayada P, Leclet H, Chopin D: Kyphoplasty. Joint Bone Spine 2002; 69: 256-261.

7. Ledlie JT, Renfro M: Balloon kyphoplasty: one-year outcomes in vertebral body height restoration, chronic pain, and activity levels. J Neurosurg 2003; 98: 36-42.

8. Hulme PA, Krebs J, Ferguson SJ, Berlemann U: Vertebroplasty and Kyphoplasty: a systematic review of 69 clinical studies. Spine 2006; 31: 1983-2001.

9. Lin EP, Ekholm S, Hiwatashi A, Westesson PL: Vertebroplasty: cement leakage into the disc increases the risk of new fracture of adjacent vertebral body. AJNR Am J Neuroradiol 2004; 25: 175-180.

10. Truumees E, Hilibrand A, Vaccaro AR: Percutaneous vertebral augmentation. Spine J 2004; 4: 218-229. 
11. Lyritis GP, Magiasis B, Eliopoulos A, Tsekoura M, Ioakimidis D: Analgesic effect of salmon calcitonin in cases of osteoporotic vertebral fractures. (in Christiansen C, Overgaard K eds. Osteoporosis. Copenhagen, Osteopress: 1392-1395, 1990).
12. Gennari C, Agnusdei D, Camporeale A: Use of calcitonin in the treatment of bone pain associated with osteoporosis. Calcif Tissue Int 1991; 49: S9-13.

13. Silverman SL: The clinical consequences of vertebral compression fracture. Bone 1992; 13: S27-31. 